Results Baseline characteristics and the prevalence of cardiovascular risk factors were similar between Groups A and B (Table 1). The rate of progression to ICA was comparable in the two groups $(9.9 \%$ vs $12.0 \% ; \mathrm{p}=0.377)$, as was the rate of revascularization $(4.0 \%$ vs $5.0 \% ; \mathrm{p}=0.532)$ (Figure 1$)$. The average per investigated patient cost was lower in Group A by $£ 46.11$ ( $£ 279.66$ vs $£ 325.77$ ).

In Group A1 there was a lower rate of progression to ICA compared to Group B1 $(8.7 \%$ vs $12.6 \%, \mathrm{p}=0.177)$ as was for revascularization $(2.6 \%$ vs $5.5 \%, \mathrm{p}=0.122)$. The average per patient cost was considerably lower in Group A1 by $£ 69.54$.

$18.5 \%$ of patients proceeded to ICA in Group A2 compared to $14.6 \%$ in Group B2 $(\mathrm{p}=0.512)$ and $10.8 \%$ were revascularized as opposed to $5.2 \%(\mathrm{p}=0.187)$. The average cost per investigated patient was slightly higher in Group A2 by $£ 20.99$.

Investigating possible predictors of revascularization (ESC RS, diabetes, family history of coronary artery disease, smoking, hypercholesterolaemia), only the ESC RS was found to be independently related to the need for revascularization (OR: 1.049, 95\%CI: 1.036-1.062, $\mathrm{p}<0.001)$.

Conclusion Both NICE and ESC recommendations on new onset stable chest pain lead to similar rates of progression to ICA and revascularization, but lower cost when stress echo (ESC guidance) is used as first line investigation. There was no significant difference when we repeated the analysis in low and high ESC RS patients. However, the ESC RS was the only independent predictor of need for revascularization.

Conflict of Interest None

\section{CORNEAL BIOMECHANICAL PROPERTIES AND VASCULAR COMPLIANCE IN THE UK BIOBANK COHORT}

${ }^{1}$ Simon Woodbridge*, ${ }^{2}$ Swan Kang, ${ }^{3}$ Nay Aung, ${ }^{4}$ Luca Biasiolli, ${ }^{5}$ Jackie Cooper, ${ }^{3}$ Mihir Sanghvi, ${ }^{5}$ Kenneth Fung, ${ }^{4}$ Stefan Piechnik, ${ }^{6}$ Stefan Neubauer, ${ }^{5}$ Steffen Petersen, ${ }^{7}$ Paul Foster. ${ }^{1}$ William Harvey Research Institute; ${ }^{2}$ Moorfields Eye Hospital NHS Foundation Trust, London, UK; ${ }^{3}$ William Harvey Research Institute, Queen Mary University of London; ${ }^{4}$ Division of Cardiovascular Medicine, University of Oxford; ${ }^{5}$ William Harvey Research Institute, NIHR Barts Biomedical Research Centre, London, UK; ${ }^{6}$ Oxford University; ${ }^{7}$ NIHR Biomedical Research Centre, Moorfields Eye Hospital NHS Foundation Trust and UCL Institute of Ophthalmology, London

\subsection{6/heartjnl-2019-BCS.107}

Introduction Intra-ocular pressure (IOP) measurement is an integral part a comprehensive eye examination. In addition to IOP, corneal biomechanical characteristics such as corneal hysteresis $(\mathrm{CH})$, a measurement of viscoelastic compliance, and corneal resistance factor (CRF), derived from corneal deformability, have also been identified as useful indicators of incidence and progression of primary open angle glaucoma (POAG) $(1,2)$. Corneal tissue shares compositionally similar properties with arterial tissue $(3,4)$. Our cross-sectional observational study aimed to ascertain whether corneal biomechanical metrics $(\mathrm{CH} \&$ CRF) are associated with arterial stiffness - a well-established marker of future cardiovascular (CV) events and mortality.

Methods From an initial pool of 5065 participants from the community-based UK Biobank study, 4018 were rejected for missing data, leaving a cohort of 1047 individuals (male/female ratio: 0.496, mean age: 62 years, white ethnicity: 96.1\%) (Table 1). Corneal biomechanical metrics ( $\mathrm{CH} \& \mathrm{CRF}$ ), were obtained using a Reichert Ocular Response Analyzer (ORA). Arterial compliance was quantified by aortic distensibility (AoD) derived by cardiovascular magnetic resonance (CMR) imaging. The relationship between corneal and vascular compliance parameters was assessed using both Spearman rank correlation coefficient analysis, and univariable and multivariable regression analyses adjusting for potential influential confounding variables - age, sex, ethnicity, height, weight, systolic blood pressure (SBP), diastolic blood pressure (DBP), smoking status, regular alcohol intake, diabetes status and dyslipidaemia.

Results A significant weakly positive correlation was observed between $\mathrm{CH}$ and $\mathrm{AoD}$ at both the ascending aorta (AA) and proximal descending aorta (PDA) (AA: Rho $=0.08, \mathrm{p}=0.01 ;$ PDA: Rho $=0.11, \mathrm{p}<0.01)$, however no significant correlation was observed between CRF and AoD.

In univariable analysis, only $\mathrm{CH}$ produced significant changes in AoD at both the AA and PDA (AA: $B=+3.0 \%$ per $10 \%$ increase in $\mathrm{CH}, 95 \% \mathrm{CI}=0.6$ to $5.5, \mathrm{p}=0.02$; PDA: $\beta=+2.6 \%$ per $10 \%$ increase in $\mathrm{CH}, 95 \% \mathrm{CI}=0.8$ to $4.4, p=0.004$ ) (Figure 1 ). There was no significant linear relationship between $\mathrm{CH}$ or $\mathrm{CRF}$ and $\mathrm{AoD}$ in multivariable regression analysis, at both the $\mathrm{AA}$ and $\mathrm{PDA}(\mathrm{CH}$ at $\mathrm{AA}: \mathrm{B}=+0.8 \%$ per $10 \%$ increase in $\mathrm{CH}, 95 \% \mathrm{CI}=-0.9$ to 2.6, $\mathrm{p}=0.37 ; \mathrm{CH}$ at $\mathrm{PDA}: \beta=+0.8 \%$ per $10 \%$ increase in $\mathrm{CH}, 95 \% \mathrm{CI}=-0.3$ to $2.0, \mathrm{p}=0.16$; $\mathrm{CRF}$ at $\mathrm{AA}: ß=+1.3 \%$ per $10 \%$ increase in $\mathrm{CH}, 95 \% \mathrm{CI}=-0.4$ to $3.0, p=0.13$; $\mathrm{CRF}$ at $\mathrm{PDA}: \beta=+0.9 \%$ per $10 \%$ increase in $\mathrm{CH}, 95 \% \mathrm{CI}=-0.3$ to $2.0, \mathrm{p}=0.13$ ).

Conclusion In this community-based cohort, we observed a weakly significant general correlation between $\mathrm{CH}$ and AoD. After adjustment for potential confounding factors, we then observed no significant relationship between corneal and aortic biomechanical indices, suggesting that in a general population, biomechanical corneal indices are not independently associated with parameters of central arterial compliance.

Conflict of Interest None 